\title{
Cardiovascular magnetic resonance-derived left ventricular mechanics-strain, cardiac power and end-systolic elastance under various inotropic states in swine
}

\author{
A. Faragli 1,2,3,4, R. Tanacli 1,4, C. Kolp ${ }^{1}$, D. Abawi ${ }^{1}$, T. Lapinskas ${ }^{4,5}$, C. Stehning ${ }^{6}$, B. Schnackenburg ${ }^{6}$, F. P. Lo Muzio ${ }^{7,8}$, \\ L. Fassina ${ }^{9}$, B. Pieske ${ }^{1,2,3,4}$, E. Nagel ${ }^{10}$, H. Post ${ }^{1,2,3,11}$, S. Kelle ${ }^{1,2,3,4+}$ and A. Alogna ${ }^{1,2,3^{*+}}$
}

\begin{abstract}
Background: Cardiovascular magnetic resonance (CMR) strain imaging is an established technique to quantify myocardial deformation. However, to what extent left ventricular (LV) systolic strain, and therefore LV mechanics, reflects classical hemodynamic parameters under various inotropic states is still not completely clear. Therefore, the aim of this study was to investigate the correlation of LV global strain parameters measured via CMR feature tracking (CMR-FT, based on conventional cine balanced steady state free precession (bSSFP) images) with hemodynamic parameters such as cardiac index (CI), cardiac power output (CPO) and end-systolic elastance (Ees) under various inotropic states.

Methods: Ten anaesthetized, healthy Landrace swine were acutely instrumented closed-chest and transported to the CMR facility for measurements. After baseline measurements, two steps were performed: (1) dobutamine-stress (Dobutamine) and (2) verapamil-induced cardiovascular depression (Verapamil). During each protocol, CMR images were acquired in the short axisand apical $2 \mathrm{Ch}, 3 \mathrm{Ch}$ and $4 \mathrm{Ch}$ views. MEDIS software was utilized to analyze global longitudinal (GLS), global circumferential (GCS), and global radial strain (GRS).

Results: Dobutamine significantly increased heart rate, $\mathrm{Cl}, \mathrm{CPO}$ and Ees, while Verapamil decreased them. Absolute values of GLS, GCS and GRS accordingly increased during Dobutamine infusion, while GLS and GCS decreased during Verapamil. Linear regression analysis showed a moderate correlation between GLS, GCS and LV hemodynamic parameters, while GRS correlated poorly. Indexing global strain parameters for indirect measures of afterload, such as mean aortic pressure or wall stress, significantly improved these correlations, with GLS indexed for wall stress reflecting LV contractility as the clinically widespread LV ejection fraction.

Conclusion: GLS and GCS correlate accordingly with LV hemodynamics under various inotropic states in swine. Indexing strain parameters for indirect measures of afterload substantially improves this correlation, with GLS being as good as LV ejection fraction in reflecting LV contractility. CMR-FT-strain imaging may be a quick and promising tool to characterize LV hemodynamics in patients with varying degrees of LV dysfunction.
\end{abstract}

\footnotetext{
*Correspondence: alessio.alogna@charite.de

${ }^{\dagger}$ S. Kelle and A. Alogna contributed equally to this work

${ }^{1}$ Department of Internal Medicine and Cardiology, Charité-

Universitätsmedizin Berlin, Campus Virchow-Klinikum, Augustenburger

Platz 1, 13353 Berlin, Germany

Full list of author information is available at the end of the article
}

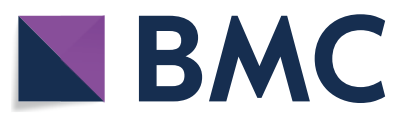

(c) The Author(s) 2020. Open Access This article is licensed under a Creative Commons Attribution 4.0 International License, which permits use, sharing, adaptation, distribution and reproduction in any medium or format, as long as you give appropriate credit to the original author(s) and the source, provide a link to the Creative Commons licence, and indicate if changes were made. The images or other third party material in this article are included in the article's Creative Commons licence, unless indicated otherwise in a credit line to the material. If material is not included in the article's Creative Commons licence and your intended use is not permitted by statutory regulation or exceeds the permitted use, you will need to obtain permission directly from the copyright holder. To view a copy of this licence, visit http://creativecommons.org/licenses/by/4.0/. The Creative Commons Public Domain Dedication waiver (http://creativeco mmons.org/publicdomain/zero/1.0/) applies to the data made available in this article, unless otherwise stated in a credit line to the data. 
Keywords: Cardiovascular magnetic resonance, Feature tracking, Left ventricular strain, Contractile function, Porcine model, Translational studies, Hemodynamics

\section{Background}

The routine assessment of left ventricular (LV) ejection fraction (LVEF), being a measure of global systolic function, falls short in identifying regional myocardial impairment and the mechanical contraction of the heart $[1,2]$. Therefore, strain imaging has emerged in the past years to better quantify myocardial LV deformation in various patient populations [3-7]. Numerous studies have validated and shown the utility of myocardial strain in the diagnosis of several pathologies, identifying subclinical myocardial changes, and even by showing an impact on the prognosis of cardiovascular pathologies [8-15]. Recently, cardiovascular magnetic resonance (CMR) feature tracking (FT) strain analysis was shown to be accurate in the detection of myocardial dysfunction as well as useful as a predictor of major adverse cardiac events, with the advantage of utilizing conventional balanced steady-state free precession (bSSFP) cine sequences [1519]. Impaired LV systolic function and cardiac reserve can be clinically assessed by hemodynamic parameters such as cardiac index (CI), as well as by cardiac power output $(\mathrm{CPO})$ at rest and during pharmacological stress. In particular, the latest has been shown to strongly correlate with the clinical outcome of chronic heart failure patients [20,21]. Moreover, LV CPO is able to provide an assessment of the intraventricular flow as well as of its mechanics much more than other hemodynamic parameters, since it couples not only with the cardiac work, but also with the response of the vasculature [22, 23]. The invasively measured end-systolic elastance (Ees) is instead, a relatively load-independent parameter describing the LV inotropic state [24]. Recently, Seeman and colleagues successfully investigated a novel CMR method to noninvasively quantify Ees [25]. Whether or not, and to what extent CMR-FT LV strain reflects the above-mentioned hemodynamic parameters under various inotropic states has not been investigated thus far. Therefore, the aim of this study was to validate the correlation of CMR LV strain parameters against hemodynamic parameters such as $\mathrm{CI}, \mathrm{CPO}$ and the Ees mentioned above, under various inotropic states in swine.

\section{Methods}

The experimental protocols were approved by the local bioethics committee of Berlin, Germany (G0138/17), and conform to the "European Convention for the Protection of Vertebrate Animals used for Experimental and other
Scientific Purposes" (Council of Europe No 123, Strasbourg 1985).

\section{Experimental setup}

Female Landrace swine $(\mathrm{n}=10,51 \pm 10 \mathrm{~kg})$ were fasted overnight with free access to water, and then sedated and intubated on the day of the experiment. Anaesthesia was continued with fentanyl, midazolam, ketamine and pancuronium as needed. The anesthesia regimen included low-dose isofluorane to obtain a deeper sedation and stabilize hemodynamics without impacting much on systemic vascular resistance. Animals were ventilated (Cato, Dräger Medical, Lubeck, Germany) with a $\mathrm{FiO} 2$ of 0.5, an I: E-ratio of 1:1.5, the positive end-expiratory pressure was set at $5 \mathrm{mmHg}$ and a tidal volume of $10 \mathrm{ml} \mathrm{kg}^{-1}$. The respiratory rate was continuously adjusted to maintain an end-expiratory carbon dioxide partial pressure between 35 and $45 \mathrm{mmHg}$. Under fluoroscopic guidance, all animals were instrumented with a floating balloon catheter in the right atrium, as well as in the coronary sinus (Arrow Balloon Wedge-Pressure Catheters, Teleflex Inc, Wayne, Pennsylvania USA). In order to avoid CMRartefacts, the balloon-tip was cut before introducing the catheters in the vessel. Respiratory gases (PM 8050 MRI, Dräger Medical), heart rate (HR), and invasively derived arterial blood pressure were continuously monitored (Precess 3160, InVivo, Gainesville, Florida, USA) via a sheath access surgically prepared in the internal carotid artery. Body temperature was monitored by a sublingual thermometer and was maintained at $38{ }^{\circ} \mathrm{C}$ during CMR imaging via air ventilation or infusion of cold saline solution.

\section{Experimental protocols}

After acute instrumentation, the animals were transported to the CMR facility for measurements. After baseline measurements, two steps were performed: (1) Dobutamine-stress (Dobutamine) and (2) verapamilinduced cardiovascular depression (Verapamil). Dobutamine infusion was titrated aiming at a $25 \% \mathrm{HR}$ increase compared to baseline values, while verapamil was given as single $2.5 \mathrm{mg}$ bolus, aiming at a $25 \%$ decrease of CI. This protocol was established beforehand with a small pilot study (data not shown), in which the titration of dobutamine and verapamil was assessed by LV invasive conductance measurements according to previous publications by our group [26]. The cumulative dose for each experiment was achieved via careful titration of 
verapamil, administered as a $2.5 \mathrm{mg}$ bolus in order to avoid a pronounced hypotension leading to hemodynamic instability. In the pilot experiments CI was continuously assessed online via a Swan-Ganz catheter in the pulmonary artery (Edwards Lifesciences CCO connected to Vigilance I, Edwards Lifesciences, Irvine, California, USA). In the CMR study, after the first bolus, we estimated stroke volume via short-axis cine imaging after reaching a hemodynamic steady state (around $5 \mathrm{~min}$ after bolus injection). In case CI was not decreased as much as $25 \%$, we proceeded with a further bolus of verapamil. Between the different protocol steps there was a washout period of $30 \mathrm{~min}$. The anaesthesia regimen included low dose isoflurane to obtain a deeper sedation and stabilize hemodynamics without impacting much on systemic vascular resistance. This protocol was established beforehand with a small pilot study (data not shown), in which the titration of dobutamine and verapamil was assessed by LV invasive conductance measurements according to previous publications by our group [26]. At each protocol step, CMR images were acquired in the short axis (SAx), two-chamber $(2 \mathrm{Ch})$, three-chamber $(3 \mathrm{Ch})$ and fourchamber (4Ch) views. At the end of the measurements, animals were transported back to the operating room for sacrifice. Sacrifice was performed with an intracoronary $80 \mathrm{mmol}$ potassium bolus.

\section{Cardiovascular magnetic resonance}

All CMR images were acquired in a supine position using a 3 T CMR scanner (Ingenia, Philips Healthcare, Best, The Netherlands) CMR scanner with an anterior- and a built-in posterior coil element, where up to 30 coil elements were employed, depending on the individual anatomy. All animals were scanned using identical comprehensive imaging protocol. The study protocol included initial scouts to determine cardiac imaging planes. Cine images were acquired using electrocardiogram (ECG)gated bSSFP cine sequence in three LV long-axis (2Ch, $3 \mathrm{Ch}, 4 \mathrm{Ch}$ ) planes. The ventricular $2 \mathrm{Ch}$ and $4 \mathrm{Ch}$ planes were used to plan stack of SAx slices covering entire LV. The following imaging parameters were used: repetition time $(\mathrm{TR})=2.9 \mathrm{~ms}$, echo time $(\mathrm{TE})=1.45 \mathrm{~ms}$, flip angle $=45^{\circ}$, measured voxel size $=1.9 \times 1.9 \times 8.0 \mathrm{~mm}^{3}$, reconstructed voxel size $1.0 \times 1.0 \times 8 \mathrm{~mm}^{3}$, and 40 cardiac phases.

\section{Image analysis}

All images were analyzed offline using a commercially available software (Medis Suite, version 3.1, Leiden, The Netherlands) in accordance with a recent consensus document for quantification of LV function using CMR [27]. A numeric code was assigned to the sequences of different measurements steps and the observers were therefore blinded to the pharmacological interventions. Given the excellent inter-observer reproducibility, we averaged values obtained by several measurements from one observer.

On SAx view, the outline of the endocardial border of the LV was manually traced on all slices of each phase. Volumes were computed by Simpson method of disks summation, whereby the sum of cross-sectional areas was multiplied by slice thickness $(8 \mathrm{~mm})$. The LVEF was calculated using the Simpson method. The LV outflow tract was included as LV blood volume. Papillary muscles and trabeculation were included as LV volume. The ascending aorta was outlined in all the images and flow calculation was performed in the corresponding velocityencoded phase images. The average flow velocity $(\mathrm{cm} / \mathrm{s})$ was multiplied by the area of the vessel $\left(\mathrm{cm}^{2}\right)$ to obtain the flow $(\mathrm{ml} / \mathrm{s})$ and integrated over one cardiac cycle to obtain the stroke volume (SV). Then, the cardiac output (CO) is indirectly calculated as the product of SV and $\mathrm{HR}$. Finally, the $\mathrm{CI}$ is calculated as the $\mathrm{CO}$ divided by the body surface area (BSA) [28]. For the strain analysis $2 \mathrm{Ch}, 3 \mathrm{Ch}$ and $4 \mathrm{Ch}$ cine images, and respectively, 3 preselected mid-ventricle slices from the LV SAx stack were included. The endocardial and epicardial contours drawn on cine images with QMass (version 8.1, Medis Medical) were transferred to QStrain RE (version 2.0, Medis Medical) where after the application of tissue tracking algorithm, endocardial and epicardial borders were detected throughout all the cardiac cycle (Fig. 1a, d). These longaxis cine images were further used to compute myocardial global longitudinal strain (GLS), and SAx images were used to compute global circumferential strain (GCS) and global radial strain (GRS) and strain-rate (Fig. 1b, e). The global values were obtained by averaging the values of systolic peak strain according to an AHA 17 segments model, apex being excluded, as follows: GCS from averaging circumferential strain for 6 basal, $6 \mathrm{mid}$ and 4 apical segmental individual values; GLS from $2 \mathrm{Ch}, 3 \mathrm{Ch}$ and 4Ch averaging 6 basal, 6 mid and 4 apical segments using a bull-eye view (Fig. 1c, f, g, h, i). Data on strain rate are presented in Table 3. In line with the global strain parameters, dobutamine increased peak systolic SR. Verapamil significantly decreased peak systolic SR compared to dobutamine but not to baseline values.

\section{Hemodynamic parameters}

Systolic blood pressure (SBP), diastolic blood pressure (DBP) and mean aortic pressure (mAoP) were invasively measured throughout the entire protocol study via a sheath access surgically prepared in an internal carotid artery.

The systemic vascular resistance was calculated as follows: 


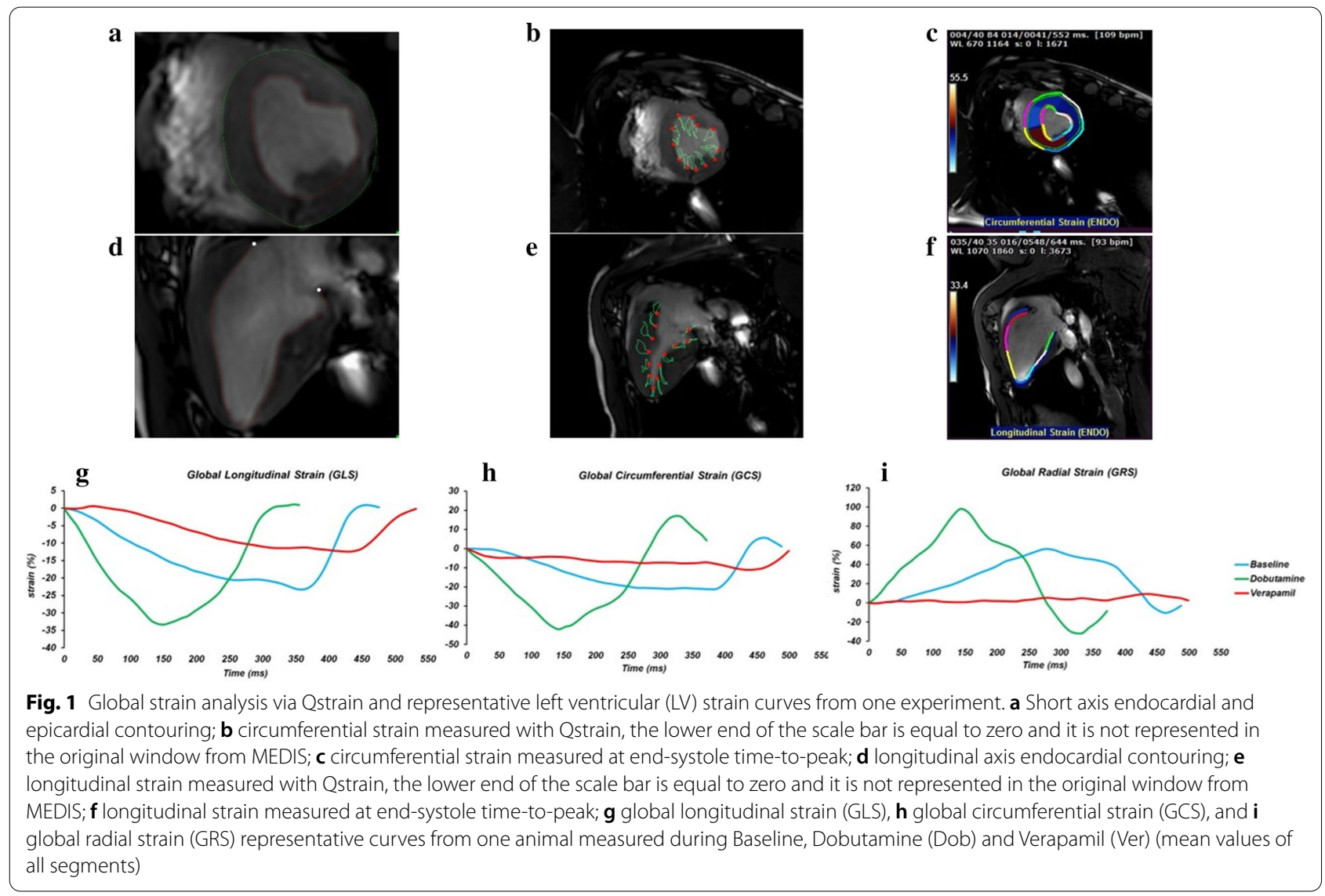

$$
S V R_{m m H g} / L=\frac{\text { Mean Arterial Pressure }(M A P)-\text { Right Atrial Pressure }}{\text { Cardiac Output }(C O)}
$$

$\mathrm{CPO}, \mathrm{CI}$ and Ees were calculated as follows:

$$
\begin{aligned}
& C P O=\frac{C O \times m A o P}{451} \\
& C I=\frac{C O}{B S A} \\
& E e s=\frac{L V P_{\text {sys }}}{V_{L V P_{\max }}-V_{0}}
\end{aligned}
$$

where:

$$
V_{0}=0
$$

as described in the work by Kelly et al [29].

GLS, GCS and GRS were indexed to the measured mAoP adapting the formula from the previous study by Rhea et al. [30] as follows:

\section{Global Strain $\times m A o P$ $\operatorname{avg}(m A o P)$}

where Global Strain was the global value of either longitudinal (GLSi), circumferential (GCSi) or radial (GRSi) strain and $\operatorname{avg}(\mathrm{mAoP})$ was the average of the $\mathrm{mAoP}$

$L V P_{\text {sys }}=\frac{2}{3}$ Systolic Blood Pressure $+\frac{1}{3}$ Diastolic Blood Pressure

$V_{L V P_{\max }}=$ End - Systolic Volume measured for each protocol step, namely at baseline, dobutamine and verapamil (Table 1). 
Table 1 Systemic hemodynamics and cardiac mechanics parameters during BL, Dob and Ver steps

\begin{tabular}{lccl}
\hline & Baseline & Dobutamine & Verapamil \\
\hline $\mathrm{HR}(\mathrm{bpm})$ & $106 \pm 15$ & $146 \pm 12^{*}$ & $98 \pm 19^{*, \$}$ \\
$\mathrm{LVEF}(\%)$ & $59 \pm 8$ & $77 \pm 7^{*}$ & $39 \pm 9^{*, \$}$ \\
$\mathrm{CO}(\mathrm{L} / \mathrm{min})$ & $6 \pm 1$ & $9 \pm 2^{*}$ & $4 \pm 1^{*, \$}$ \\
$\mathrm{Cl}\left(\mathrm{L} / \mathrm{min} / \mathrm{m}^{2}\right)$ & $2.5 \pm 0.2$ & $3.8 \pm 0.5^{*}$ & $1.7 \pm 0.7^{*, \$}$ \\
$\mathrm{CPO}(\mathrm{W})$ & $1.2 \pm 0.3$ & $2.0 \pm 0.6^{*}$ & $0.7 \pm 0.2^{*, \$}$ \\
$\left.\mathrm{SVR}(\mathrm{dyn} \mathrm{s} \mathrm{cm})^{-5}\right)$ & $15 \pm 5$ & $11 \pm 4^{*}$ & $19 \pm 9^{*, \S}$ \\
$\mathrm{mAoP}(\mathrm{mmHg})$ & $90 \pm 12$ & $98 \pm 19$ & $70 \pm 10^{*, \$}$ \\
Wall stress $(\mathrm{mmHg})$ & $0.12 \pm 0.02$ & $0.16 \pm 0.04^{*}$ & $0.10 \pm 0.02^{\S}$ \\
\hline
\end{tabular}

$H R$ heart rate, LVEFleft ventricular ejection fraction, CO cardiac output, CIcardiac index, CPO cardiac power output, SVR systemic vascular resistance, $m A o P$ mean aortic pressure

${ }^{*} p<0.05$ vs. Baseline; ${ }^{\S} p<0.05$ vs. Dobutamine

Meridional wall stress was calculated via the following formula [31]:

$$
L V \text { wall stress }=\frac{\left(0.334 \times L V P_{s y s} \times L V E S D\right)}{P W T \times[1+P W T / L V I D]}
$$

where $\mathrm{LVESD}=$ left ventricular end-systolic diameter and $\mathrm{PWT}=$ posterior wall thickness as described in the paper by Reichek et al. [31]. PLT was measured by averaging three separate measurements in the basal short axis sequence.

GLS, GCS and GRS were indexed to the measured wall stress adapting the formula from the study by Reichek et al. [31] as follows:

$$
\frac{\text { Global Strain } \times L V \text { wall stress }}{\operatorname{avg}(L V \text { wall stress })}
$$

where Global Strain was the global value of either global longitudinal (GLSw), global circumferential (GCSw) or global radial (GRSw) strain. The average for both LV wall stress were calculated for each step, namely at baseline, dobutamine and verapamil (Table 1).

\section{Statistical analysis}

All data are presented as mean $\pm \mathrm{SD}$. The association between strain data and hemodynamic data was assessed by linear regression analysis. The condition (baseline, dobutamine, verapamil) was included as a regressor into the linear regression model. To verify whether the linear regressions were significantly different ( $p$-value $<0.05$ ), using custom-made scripts in MATLAB (release R2020a; The MathWorks, Inc., Natick, Massachusetts, USA), we compared slopes, intercepts as well as correlation coefficients: (i) via t-test (for slopes and intercepts) and (ii) via the Fisher's r-to-z transformation followed by $z$-test (for correlation coefficients), as previously described by Weaver and Wuensch [32]. Data between groups at different inotropic states were analysed by one-way ANOVA for repeated measurements. Post-hoc testing was performed by Tukey's test. A p-value $<0.05$ was considered significant. For statistical calculations, we used the software Sigmastat (Version 4.0, Systat Software, Inc., Cranes Software, Karnataka, India) and SPSS (Version 23.0, Statistical Package for the Social Sciences, International Business Machines, Inc., Armonk, New York, USA).

\section{Results}

The dose of dobutamine needed to induce a $25 \% \mathrm{HR}$ increase was $6.4 \pm 2.5 \mu \mathrm{g} / \mathrm{kg} / \mathrm{min}$, while the dose of verapamil needed to decrease CI significantly was $5 \pm 2 \mathrm{mg}$.

\section{Systemic hemodynamics}

Systemic hemodynamic data are summarized in Table 1. mAoP was not affected by Dobutamine, but significantly decreased during Verapamil. Dobutamine increased baseline HR, CO and LVEF, while Verapamil decreased them. Systemic vascular resistance (SVR) substantially decreased during Dobutamine, while increased during Verapamil. CPO and CI both increased during Dobutamine and decreased during Verapamil. Ees, the slope of the end-systolic pressure-volume relationship, increased during Dobutamine and decreased during Verapamil infusion (Fig. 2).

\section{Global strain parameters}

Strain parameters are summarized in Table $2 \mathrm{a}, \mathrm{b}$ and c. GLS as well as GCS increased during Dobutamine, while decreased during Verapamil. GRS was not significantly affected by Dobutamine, while decreased significantly during Verapamil.

\section{Systolic strain rate}

Data on strain rate (SR) are presented in Table 3. In line with the global strain parameters, Dobutamine increased peak systolic SR. Verapamil significantly decreased peak systolic SR compared to Dobutamine but not to baseline values.

\section{Indexing strain parameters for indirect measures of afterload}

Indexing global strain parameters for either mAoP (Table 2b) or for meridional wall stress (Table 2c) did not significantly impact the above-described changes induced by Dobutamine and Verapamil. 


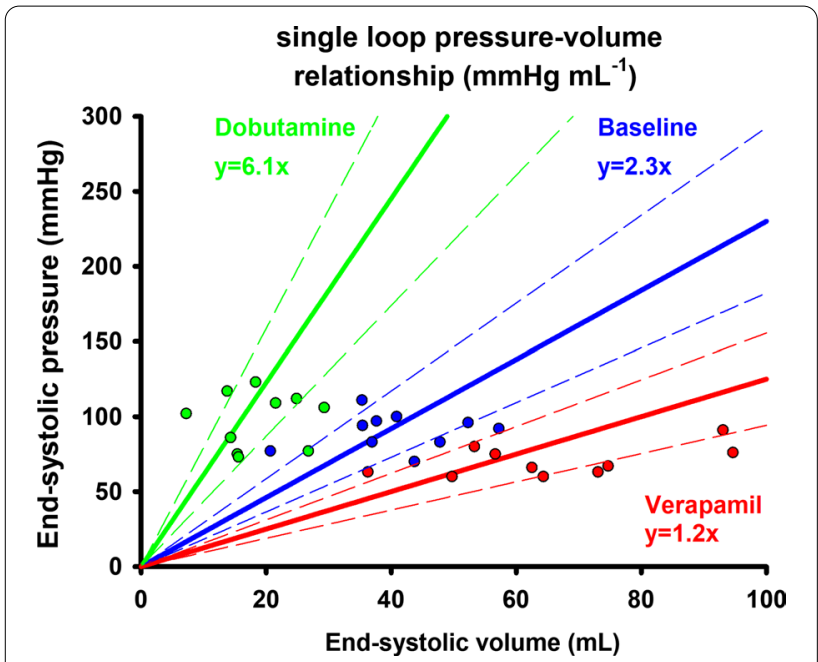

Fig. 2 Averaged end-systolic pressure-volume relationship at baseline, during dobutamine and verapamil. Single-loop derived by the LV end-systolic pressure-volume relationship (ESPVR) is plotted under various inotropic states. The green line corresponds to the averaged ESPVR during dobutamine infusion, the blue line represents the averaged ESPVR at baseline, while the red one represented the averaged ESPVR at verapamil. A steeper increase in ESPVR during dobutamine and a relevant decrease during verapamil are observed. The equation for each ESPVR is displayed in the graph. Data points are plotted for each animal during different inotropic states. The dashed lines represent the $95 \%$ confidence intervals

Table 2 Global strain and indexed global strain values measured at different inotropic states

\begin{tabular}{lccr}
\hline & Baseline & Dobutamine & Verapamil \\
\hline (A) & & & \\
GLS (\%) & $-23 \pm 4$ & $-45 \pm 9^{*}$ & $-16 \pm 3^{*, \$}$ \\
GCS (\%) & $-31 \pm 8$ & $-53 \pm 10^{*}$ & $-17 \pm 5^{*, 5}$ \\
GRS (\%) & $72 \pm 19$ & $88 \pm 36$ & $30 \pm 12^{*, \$}$ \\
(B) & & & \\
GLSi (\%) & $-23 \pm 4$ & $-45 \pm 10^{*}$ & $-16 \pm 4^{*, \$}$ \\
GCSi (\%) & $-30 \pm 8$ & $-52 \pm 8^{*}$ & $-16 \pm 5^{*, 5}$ \\
GRSi (\%) & $71 \pm 19$ & $84 \pm 23$ & $30 \pm 13^{*, \$}$ \\
(C) & & & \\
GLSW (\%) & $-23 \pm 5$ & $-44 \pm 10^{*}$ & $-16 \pm 3^{*, \$}$ \\
GCSW (\%) & $-31 \pm 9$ & $-52 \pm 13^{*}$ & $-17 \pm 17^{*, \$}$ \\
GRSW (\%) & $71 \pm 20$ & $90 \pm 54$ & $35 \pm 27^{*, \$}$ \\
\hline
\end{tabular}

(A) Global strain: GLS global longitudinal strain, GCS global circumferential strain, GRS global radial strain; (B) Global strain indexed for mean aortic pressure (mAoP): GLSiglobal longitudinal strain indexed for mAoP, GCSiglobal circumferential strain indexed for mAOP, GRSiglobal radial strain indexed for mAoP; (C) Global strain indexed for meridional wall stress: GLSw global longitudinal strain indexed for wall stress, GCSw global circumferential strain indexed for wall stress, GRSw global radial strain indexed for wall stress. ${ }^{*} \mathrm{p}<0.05$ vs. baseline; ${ }^{\varsigma} p<0.05$ vs. Dobutamine
Table 3 Global peak systolic strain rates values measured at different inotropic states

\begin{tabular}{llll}
\hline & Baseline & Dobutamine & Verapamil \\
\hline GLS peak systolic SR (s-1) & $-2.5 \pm 0.6$ & $-6.4 \pm 1.5^{*}$ & $-2.1 \pm 1.1^{\S}$ \\
GCS peak systolic SR (s-1) & $-3.2 \pm 2.2$ & $-8.7 \pm 2.5^{*}$ & $-2.0 \pm 1.3^{\S}$ \\
GRS peak systolic SR (s-1) & $2.7 \pm 1.0$ & $5.5 \pm 0.9$ & $2.2 \pm 1.5^{\S}$ \\
\hline
\end{tabular}

Strain Rate (SR). ${ }^{*} p<0.05$ vs. Baseline; ${ }^{\S} p<0.05$ vs. Dobutamine

\section{Correlation between global strain, LVEF and LV hemodynamic parameters}

Linear regression analysis showed a moderate correlation between GLS, GCS and CPO, while a poor one was observed between GRS and CPO (Fig. 3a). A similar correlation was observed between GLS, GCS and CI (Fig. 3b), with GRS worst performing. A moderate correlation was observed between GLS, GCS and Ees, while a poor one was observed between GRS and Ees (Fig. 3c). Indexing global strain parameters either for mAoP or for wall stress improved their correlations with $\mathrm{CPO}$ (Figs. 4a and 5a), with CI (Figs. 4b and 5b) as well as with Ees (Figs. $4 \mathrm{c}$ and $5 \mathrm{c}$ ). LVEF moderately correlated with CI and CPO $\left(r^{2}=0.81\right.$ and $r^{2}=0.69$, respectively) as GLSw with $\mathrm{CI}$ and $\mathrm{CPO}\left(\mathrm{r}^{2}=0.74\right.$ and $\mathrm{r}^{2}=0.72$, respectively). GLSw moderately correlated with Ees as well as LVEF with Ees $\left(r^{2}=0.74\right.$ versus $\left.r^{2}=0.74\right)$. The above-mentioned correlations were both statistically significant with a p $<0.0001$.

\section{Relative change of mechanics under various inotropic states}

In Fig. 6, we plotted the relative change of mechanics (global LV strain parameters) as well as hemodynamic parameters during Dobutamine and Verapamil in comparison to baseline.

Among global strain parameters, GLS showed a higher relative change than GRS during both Dobutamine and Verapamil, while the same was valid for GCS during dobutamine only. Moreover, the impact of Dobutamine was more prominently expressed by GLS than by $\mathrm{CI}$, while the impact of Verapamil on GRS was negligible when in comparison to CI. The impact of Dobutamine and Verapamil on the rest of the mechanic and hemodynamic parameters was comparable.

\section{Discussion}

CMR strain imaging is an established technique to quantify myocardial deformation. However, to what extent LV systolic strain, and therefore LV mechanics, reflects classical hemodynamic parameters under various inotropic states is still not completely clear. In the current study, we set out to investigate the correlation of LV global strain parameters measured via CMR-FT with hemodynamic 


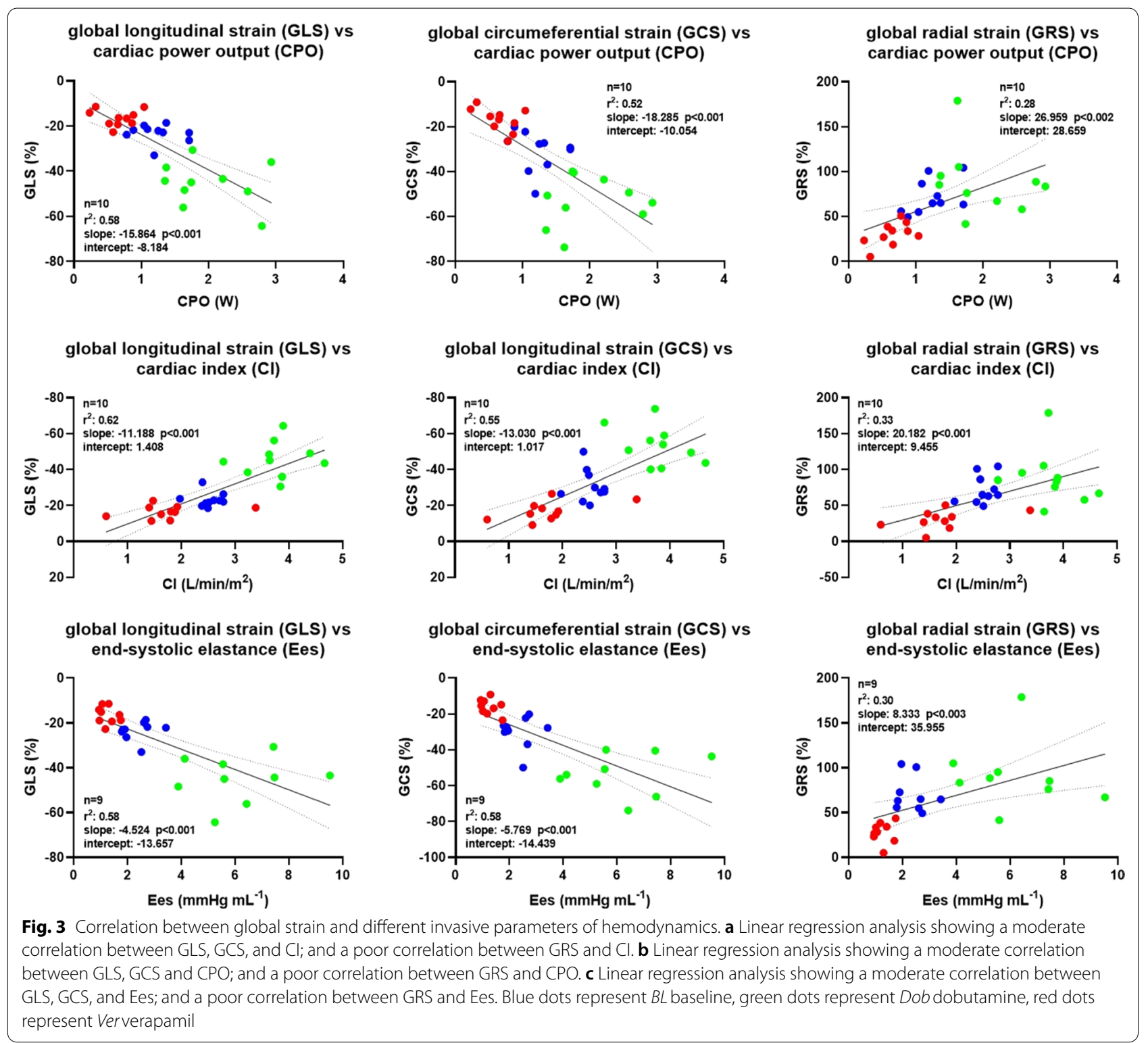

parameters under various inotropic states in swine. We observed a moderate correlation of global strain parameters with LV hemodynamics. Interestingly, indexing strain parameters for indirect measures of afterload substantially improved this correlation, with GLS indexed for wall stress reflecting LV contractility as the clinically widespread LVEF.

\section{Correlation between global strain, LVEF and LV hemodynamic parameters}

Numerous studies have reported a significant diagnostic as well as a prognostic role of LV strain in the assessment of LV mechanics in various study populations $[10$, 11 ], including patients with heart failure with preserved ejection fraction, coronary artery disease, diabetes mellitus, hypertensive heart disease, hypertrophic cardiomyopathy, and arrhythmia [1-7]. Recently, CMR-FT strain analysis was shown to be accurate in the detection of myocardial dysfunction and as well useful as a predictor of major adverse cardiac events in ischemic or nonischemic cardiomyopathy [18], with the advantage of utilizing simple bSSFP cine sequences [15-19, 33, 34]. However, notwithstanding the multitude of published papers assessing the clinical usefulness of strain, there is a notable lack of in-vivo validation studies [35]. Whether CMR-FT LV strain represents a valid tool to assess the cardiac mechanics of the myocardium, and how this varies under different inotropic states, is still unclear. In this 


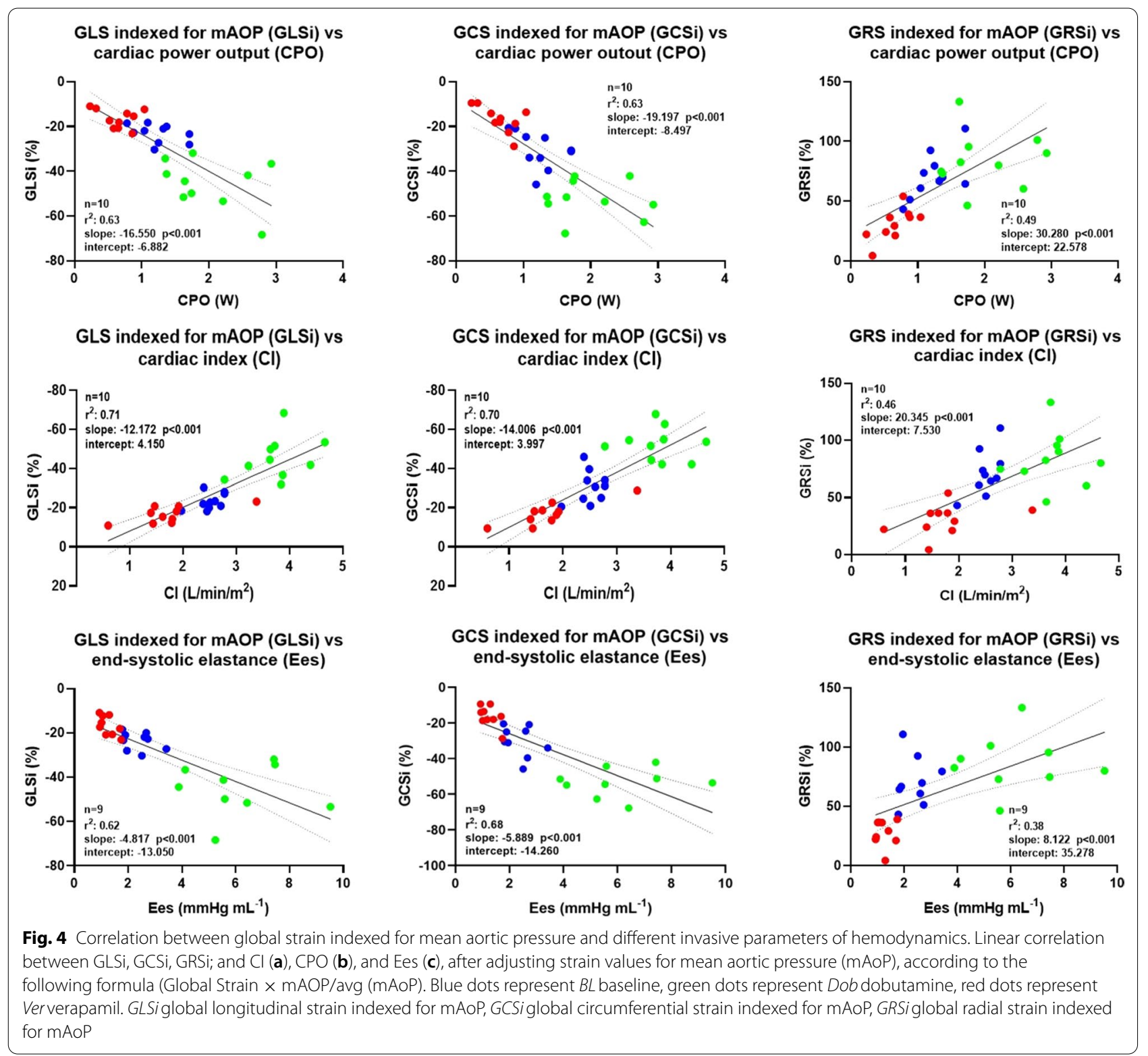

work, we could show a role of CMR LV strain as a surrogate of classic hemodynamic parameters such as $\mathrm{CI}$, $\mathrm{CPO}$, and a load-independent parameter of cardiac contractility such as Ees, which are established parameters to describe the hydraulic and mechanical role of the heart as a pump [22, 24]. Previous in vivo studies concentrated on the role of 2D and 3D speckle tracking echocardiography (STE) [35]. The main validation studies analysed the correlation of STE with the sonomicrometry technique in open-chest large animal models, showing an overall good agreement of the two techniques [35]. Similarly to our study, Weidemann et al. showed the ability of strain as well as strain rate to reflect swine LV-contractility under different inotropic states and independently of heart rate [36]. The invasively measured Ees, the slope of the endsystolic pressure-volume relationship, is a relatively loadindependent parameter describing the LV inotropic state [24]. A recent study by Seeman et al. established a reliable method to assess Ees via CMR imaging [25]. In the current work, we showed a moderate to good correlation between both GLS, GCS, and Ees. The accuracy of GLS in reflecting Ees improved when indexing for wall stress. In line with our data, Yotti et al [8] showed a moderate correlation between echocardiographic assessed GCS and invasive pressure-volume catheterization data, while on the opposite, GLS correlated poorly. The authors 


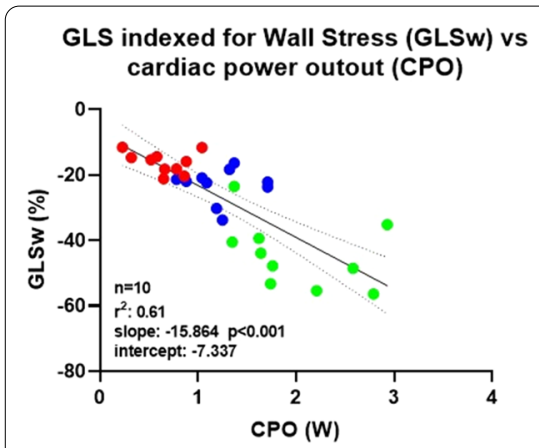

GLS indexed for Wall Stress (GLSw) vs cardiac index $(\mathrm{Cl})$

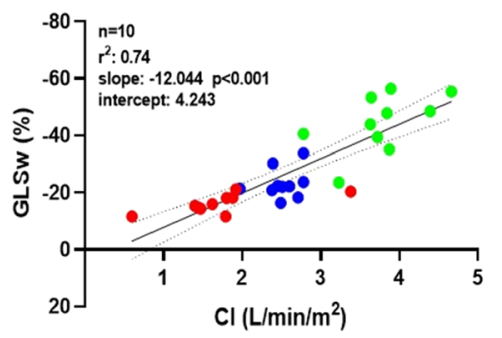

GLS indexed for Wall Stress (GLSw) vs end-systolic elastance (Ees)

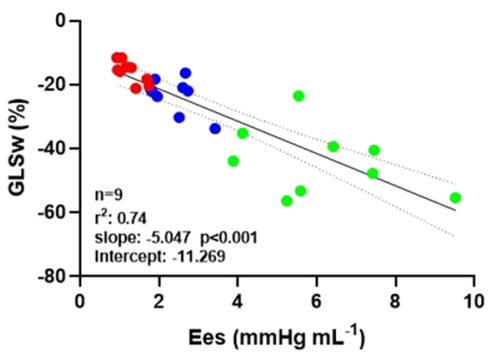

GCS indexed for Wall Stress (GCSw) vs cardiac power output (CPO)

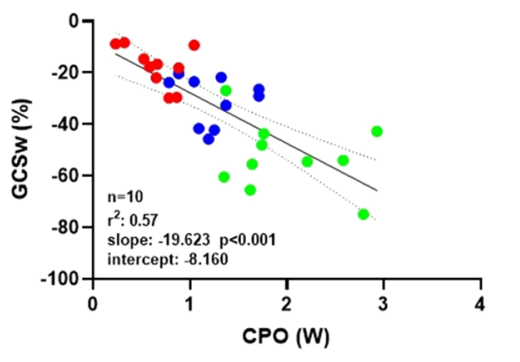

GCS indexed for Wall Stress (GCSw) vs cardiac index $(\mathrm{Cl})$

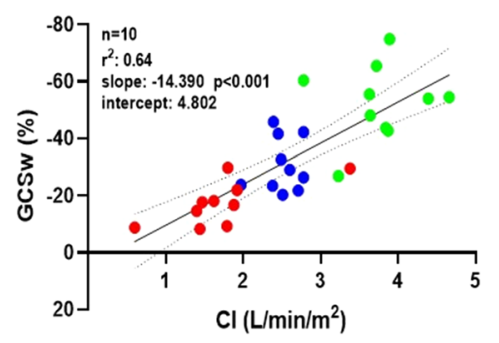

GCS indexed for Wall Stress (GCSw) vs end-systolic elastance (Ees)

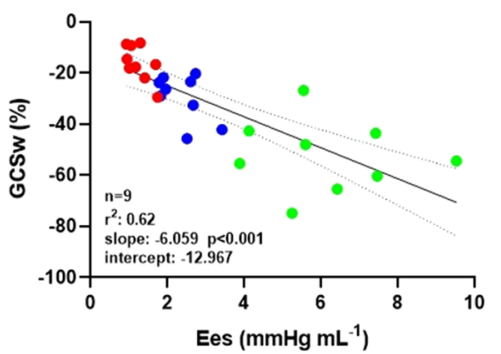

GRS indexed for Wall Stress (GRSw) vs cardiac power output (CPO)

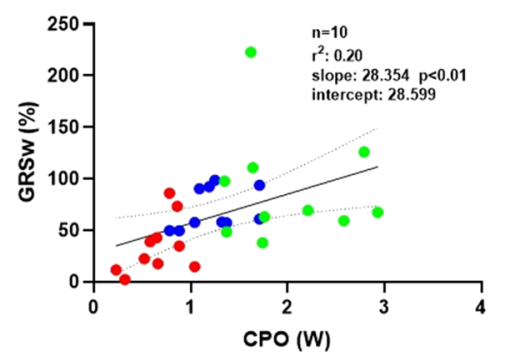

GRS indexed for Wall Stress (GRSw) vs cardiac index $(\mathrm{Cl})$

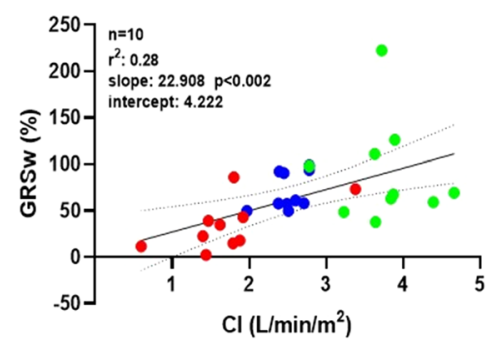

GRS indexed for Wall Stress (GRS) vs end-systolic elastance (Ees)

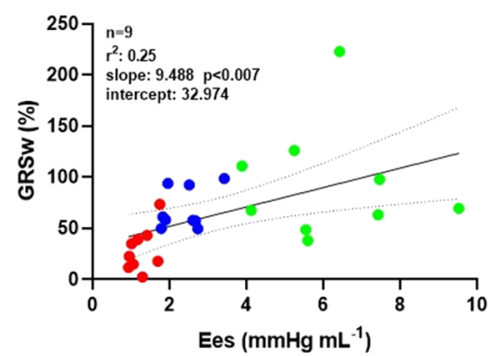

Fig. 5 Correlation between global strain indexed for wall stress and different invasive parameters of hemodynamics. Linear correlation between GLSw, GCSw, GRSw, LVEF; and CI (a), CPO (b), and Ees (c), after adjusting strain values for meridional Wall Stress, according to the following formula (Global Strain $\times$ Wall Stress / avg (Wall Stress). Blue dots represent BL baseline, green dots represent Dob dobutamine, red dots represent Ververapamil

highlighted how the GCS measurements, as opposed to the GLS, did not change in patients with aortic stenosis or hypertension. They hypothesized this could be related to a lower load-dependency [8] of the first in comparison to the latter ones, but the different methodology of strain assessment in comparison to our study is probably playing a role as well.

\section{Indexing strain parameters for indirect measures of afterload}

In the current study, in order to minimize the loaddependency of the strain measurements, we indexed all the global strain values for indirect measures of afterload, such as the invasively measured $\mathrm{mAoP}$ as well as the meridional wall stress, as already described by Rhea et al. and Reichek et al [30, 31]. Correcting the strain measurements, as mentioned above, improved the ability of strain parameters to reflect LV hemodynamics. Yingchoncharoen et al. already demonstrated that blood pressure adjustment of strain is advisable in patients with large deviations of SBP from the normalreference value [37]. Furthermore, in a study by Weiner et al. on the impact of isometric handgrip testing on LV twist mechanics, it was shown that longitudinal strain is influenced by blood pressure [38]. Finally, in line with our study, Rhea et al. showed an improved accuracy of pressure-adjusted GLS in predicting cardiac events and mortality [30]. 


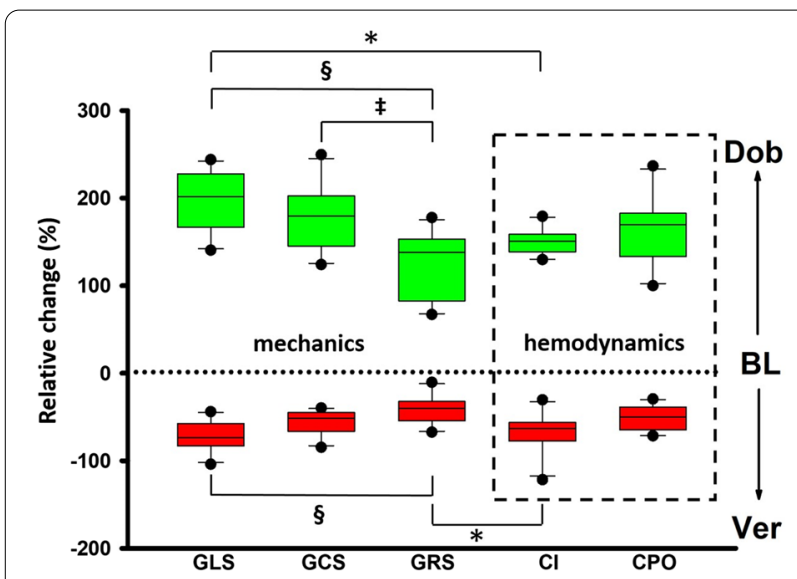

Fig. 6 Relative change of global strain and cardiac mechanics parameters from baseline to dobutamine or verapamil. The schematic representation above shows the relative change of global strain values and invasive hemodynamic parameters (dashed box) from baseline (BL, dashed horizontal line) to dobutamine (Dob, green boxes) and from BL to verapamil (Ver, red boxes), respectively. ${ }^{*} p<0.05$ vs $C l,{ }^{5} p<0.05$ vs GLS, ${ }^{\ddagger} p<0.05$ vs GCS

\section{Relative change of mechanics under various inotropic states}

In order to investigate to what extent global LV strain parameters reflect hemodynamic changes we plotted the relative change of these parameters during Dobutamine and Verapamil compared to baseline. The relative change of GLS during Dobutamine was higher than the one of CI, while the impact of Verapamil on GRS was negligible when compared to CI, overall in line with the poor reproducibility of GRS measurements. The impact of Dobutamine and Verapamil on the rest of the mechanic and hemodynamic parameters was comparable. In line with previous reproducibility studies [39-42], we showed that radial strain is a poorly reproducible and inaccurate measurement. In this study we did not show the interand intra-observer reproducibility because it was already assessed in a previous work from our group based on the same cohort [43]. Our analysis showed that measurements at baseline were good to excellent (good ICC 0.60-0.74; excellent ICC >0.74) for GLS, GCS and GRS, but that only GLS and GCS displayed good reproducibility during both dobutamine and verapamil steps, whereas radial strain was highly variable [43].

\section{Clinical and translational perspective}

In this in vivo study, we could show that CMR-FT strain parameters, such as GLS and GCS, reflect classic hemodynamic parameters such as CI, CPO, as well as a loadindependent parameter of cardiac contractility such as Ees. LV strain has indeed emerged in the past year as a valid technique to assess LV deformation with high reproducibility. However, the spread of this technique in the clinical routine is limited by the often lengthy postprocessing of the sequences, confining this important resource to the mere research field [44]. FT-strain, in particular, possesses the advantage of a quick assessment, being based on conventional bSSFP cine sequences [1519], and seems to be therefore a promising technique to allow a more extensive clinical use of LV strain. Furthermore, indexing the global strain values for indirect measures of afterload, such as the invasively measured mAoP as well as the meridional wall stress, improves the ability of strain parameters to reflect LV hemodynamics. After accounting for meridional wall stress, GLS performed as good as LVEF in reflecting LV contractility, expressed as Ees, confirming the potential role of this novel parameter in the clinical arena. These results suggest that implementing strain measurements with pressurederived variables may add accuracy to the evaluation of the mechanical and contractile function of the heart, improving the impact of LV strain in the clinical routine and helping to overcome the limitations of LVEF as a surrogate parameter of LV systolic function. In daily clinical routine, this could be potentially achieved even with a standard sphygmomanometer, as shown in the paper by Seeman at al [25].

Finally, we envision a promising role for CMR-FT LV strain investigation of chronic heart failure patients. In particular heart failure with preserved ejection fraction patients seem that they would benefit the most from this assessment, since previous studies have already shown a diagnostic and prognostic impact of strain measurements $[2]$.

\section{Limitations}

Specific limitation of the study are related to the fact that animals were investigated under general anaesthesia, in order to minimize the animals' distress and to obtain stable hemodynamic conditions. Due to easier housing and milder behaviour, all the animals were females. We therefore cannot draw any relevant conclusions on the role of gender on strain variability. A limitation regarding the strain analysis is that only endocardial values of strain were analysed. Moreover, an inter-vendor software variability should be considered if looking at the absolute strain values. Another limitation is that the animals were healthy, and even if conditions of hyper-contractility and hypo-contractility were induced, these were only transient and acutely assessed. We believe the data to be representative enough for the clinical translation, however further studies are needed in a clinical setting.

In conclusion, CMR-FT derived LV strain parameters, such as GLS and GCS, correlate accordingly with 
LV hemodynamics in swine under various inotropic states. Indexing strain parameters for indirect measures of afterload substantially improves this correlation, with GLS being as good as LVEF in reflecting LV contractility. CMR-FT strain imaging may be a quick and promising tool to characterize LV hemodynamics in patients with a various degree of LV dysfunction.

\begin{abstract}
Abbreviations
2Ch: Two chambers; 3Ch: Three chambers; 4Ch: Four chambers; BSA: Body surface area; bSSFP: Balanced steady-state free precession; Cl: Cardiac index; CMR: Cardiovascular magnetic resonance; CMR-FT: Cardiovascular magnetic resonance feature tracking; CO: Cardiac output; CPO: Cardiac power output; DBP: Diastolic blood pressure; ECG: Electrocardiogram; Ees: End-systolic elastance; ESPVR: End-systolic pressure-volume relationship; FT: Feature tracking; GCS: Global circumferential strain; GCSi: Global circumferential strain indexed for mAoP; GCSw: Global circumferential strain indexed for meridional wall stress; GLS: Global longitudinal strain; GLSi: Global longitudinal strain indexed for mAoP; GLSw: Global longitudinal strain indexed for meridional wall stress; GRS: Global radial strain; GRSi: Global radial strain indexed for mAoP; GRSw: Global radial strain indexed for meridional wall stress; HR: Heart rate; LV: Left ventricle/ left ventricular; LVEF: Left ventricular ejection fraction; LVESD: Left ventricular end-systolic diameter; LVP: Left ventricular pressure; mAoP: Mean aortic pressure; PWT: Posterior wall thickness; SAx: Short axis; SBP: Systolic blood pressure; SR: Strain rate; STE: Speckle tracking echocardiography; SVR: Systemic
\end{abstract} vascular resistance; TE: Echo time; TR: Repetition time.

\section{Aknowledgements}

Not applicable.

\section{Authors' contributions}

AA, SK, HP conceived the experiment, AF, AA, SK, CK, CS conducted the experiments, AF, RT, DA, AA, SP, CS, FPLM, LF analyzed the results. All authors revised the manuscript. All authors read and approved the final manuscript.

\section{Funding}

Open Access funding enabled and organized by Projekt DEAL. Alessandro Faragli, Heiner Post, Sebastian Kelle and Alessio Alogna received funding from DZHK (c) and by the BMBF (German Ministry of Education and Research). Sebastian Kelle is supported by a grant from Philips Healthcare.

\section{Availability of data}

Not applicable

\section{Ethics approval and consent to participate} Not applicable.

\section{Consent for publication}

Not applicable.

\section{Competing interests}

Apart from Alessandro Faragli, Heiner Post, Sebastian Kelle and Alessio Alogna, who declare to have received funding in the Funding section, none of the other authors reports a relationship with industry and other relevant entities - financial or otherwise - that might pose a conflict of interest in connection with the submitted article. The following authors report financial activities outside the submitted work: Burkert Pieske reports having received consultancy and lecture honoraria from Bayer Daiichi Sankyo, MSD, Novartis, Sanofi-Aventis, Stealth Peptides and Vifor Pharma; and editor honoraria from the Journal of the American College of Cardiology.

\section{Author details}

${ }^{1}$ Department of Internal Medicine and Cardiology, Charité-Universitätsmedizin Berlin, Campus Virchow-Klinikum, Augustenburger Platz 1, 13353 Berlin, Germany. ${ }^{2}$ Berlin Institute of Health (BIH), Berlin, Germany. ${ }^{3}$ DZHK (German Centre for Cardiovascular Research), partner site, Berlin, Germany.
${ }^{4}$ Department of Internal Medicine/Cardiology, Deutsches Herzzentrum Berlin, Augustenburger Platz 1, 13353 Berlin, Germany. ${ }^{5}$ Department of Cardiology, Medical Academy, Lithuanian University of Health Sciences, Eiveniu Street 2, 50161 Kaunas, Lithuania. ${ }^{6}$ Clinical Science, Philips Healthcare, Röntgenstr. 24, 22335 Hamburg, Germany. ${ }^{7}$ Department of Surgery, Dentistry, Paediatrics and Gynaecology, University of Verona, Via S. Francesco 22, 37129 Verona, Italy. ${ }^{8}$ Department of Medicine and Surgery, University of Parma, Via Gramsci 14, 43126 Parma, Italy. ${ }^{9}$ Department of Electrical, Computer and Biomedical Engineering (DIII), Centre for Health Technologies (CHT), University of Pavia, Via Ferrata 5, 27100 Pavia, Italy. ${ }^{10}$ Institute of Experimental and Translational Cardiac Imaging, DZHK Centre for Cardiovascular Imaging, Goethe University Hospital Frankfurt, Frankfurt am Main, Germany. ${ }^{11}$ Department of Cardiology, Contilia Heart and Vessel Centre, St. Marien-Hospital Mülheim, 45468 Mülheim, Germany.

Received: 17 November 2019 Accepted: 6 October 2020

Published online: 30 November 2020

\section{References}

1. Stanton T, Leano R, Marwick TH. Prediction of all-cause mortality from global longitudinal speckle strain: comparison with ejection fraction and wall motion scoring. Circ Cardiovasc Imaging. 2009;2:356-64.

2. Stokke TM, Hasselberg NE, Smedsrud MK, Sarvari SI, Haugaa KH, Smiseth OA, Edvardsen T, Remme EW. Geometry as a confounder when assessing ventricular systolic function: comparison between ejection fraction and strain. J Am Coll Cardiol. 2017;70:942-54

3. Kraigher-Krainer E, Shah AM, Gupta DK, Santos A, Claggett B, Pieske B, Zile MR, Voors AA, Lefkowitz MP, Packer M, McMurray JJ, Solomon SD, Investigators P. Impaired systolic function by strain imaging in heart failure with preserved ejection fraction. J Am Coll Cardiol. 2014;63:447-56.

4. Hasselberg NE, Haugaa KH, Sarvari SI, Gullestad L, Andreassen AK, Smiseth $\mathrm{OA}$, Edvardsen T. Left ventricular global longitudinal strain is associated with exercise capacity in failing hearts with preserved and reduced ejection fraction. Eur Heart J Cardiovasc Imaging. 2015;16:217-24.

5. Smedsrud MK, Sarvari S, Haugaa KH, Gjesdal O, Orn S, Aaberge L, Smiseth $\mathrm{OA}$, Edvardsen T. Duration of myocardial early systolic lengthening predicts the presence of significant coronary artery disease. J Am Coll Cardiol. 2012;60:1086-93.

6. Yang H, Sun JP, Lever HM, Popovic ZB, Drinko JK, Greenberg NL, Shiota T, Thomas JD, Garcia MJ. Use of strain imaging in detecting segmental dysfunction in patients with hypertrophic cardiomyopathy. J Am Soc Echocardiogr. 2003;16:233-9.

7. Fang ZY, Yuda S, Anderson V, Short L, Case C, Marwick TH. Echocardiographic detection of early diabetic myocardial disease. J Am Coll Cardiol. 2003:41:611-7.

8. Yotti R, Bermejo J, Benito Y, Sanz-Ruiz R, Ripoll C, Martinez-Legazpi P, del Villar CP, Elizaga J, Gonzalez-Mansilla A, Barrio A, Banares R, FernandezAviles F. Validation of noninvasive indices of global systolic function in patients with normal and abnormal loading conditions: a simultaneous echocardiography pressure-volume catheterization study. Circ Cardiovasc Imaging. 2014;7:164-72.

9. Gorcsan J 3rd, Tanaka H. Echocardiographic assessment of myocardial strain. J Am Coll Cardiol. 2011;58:1401-13.

10. Dahlslett T, Karlsen S, Grenne B, Eek C, Sjoli B, Skulstad H, Smiseth OA, Edvardsen T, Brunvand $\mathrm{H}$. Early assessment of strain echocardiography can accurately exclude significant coronary artery stenosis in suspected non-ST-segment elevation acute coronary syndrome. J Am Soc Echocardiogr. 2014;27:512-9.

11. Ng AC, Sitges M, Pham PN, da Tran T, Delgado V, Bertini M, Nucifora G, Vidaic J, Allman C, Holman ER, Bax JJ, Leung DY. Incremental value of 2-dimensional speckle tracking strain imaging to wall motion analysis for detection of coronary artery disease in patients undergoing dobutamine stress echocardiography. Am Heart J. 2009:158:836-44.

12. Nagata Y, Takeuchi M, Wu VC, Izumo M, Suzuki K, Sato K, Seo Y, Akashi YJ, Aonuma K, Otsuji Y. Prognostic value of LV deformation parameters using $2 \mathrm{D}$ and $3 \mathrm{D}$ speckle-tracking echocardiography in asymptomatic patients with severe aortic stenosis and preserved LV ejection fraction. JACC Cardiovasc Imaging. 2015;8:235-45. 
13. Rhea IB, Uppuluri S, Sawada S, Schneider BP, Feigenbaum H. Incremental prognostic value of echocardiographic strain and its association with mortality in cancer patients. J Am Soc Echocardiogr. 2015;28:667-73.

14. Buss SJ, Emami M, Mereles D, Korosoglou G, Kristen AV, Voss A, Schellberg D, Zugck C, Galuschky C, Giannitsis E, Hegenbart U, Ho AD, Katus HA, Schonland SO, Hardt SE. Longitudinal left ventricular function for prediction of survival in systemic light-chain amyloidosis: incremental value compared with clinical and biochemical markers. J Am Coll Cardiol. 2012;60:1067-76.

15. Eitel I, Stiermaier T, Lange T, Rommel KP, Koschalka A, Kowallick JT, Lotz J, Kutty S, Gutberlet M, Hasenfuss G, Thiele H, Schuster A. Cardiac magnetic resonance myocardial feature tracking for optimized prediction of cardiovascular events following myocardial infarction. JACC Cardiovasc Imaging. 2018;11:1433-44.

16. Onishi T, Saha SK, Delgado-Montero A, Ludwig DR, Onishi T, Schelbert EB, Schwartzman D, Gorcsan J 3rd. Global longitudinal strain and global circumferential strain by speckle-tracking echocardiography and featuretracking cardiac magnetic resonance imaging: comparison with left ventricular ejection fraction. J Am Soc Echocardiogr. 2015;28:587-96.

17. Mangion K, Carrick D, Carberry J, Mahrous A, McComb C, Oldroyd KG, Eteiba H, Lindsay M, McEntegart M, Hood S, Petrie MC, Watkins S, Davie A, Zhong X, Epstein FH, Haig CE, Berry C. Circumferential strain predicts major adverse cardiovascular events following an acute ST-segmentelevation myocardial infarction. Radiology. 2018. https://doi.org/10.1148/ radiol.2018181253.

18. Romano S, Judd RM, Kim RJ, Kim HW, Klem I, Heitner JF, Shah DJ, Jue J, White BE, Indorkar R, Shenoy C, Farzaneh-Far A. Feature-tracking global longitudinal strain predicts death in a multicenter population of patients with ischemic and nonischemic dilated cardiomyopathy incremental to ejection fraction and late gadolinium enhancement. JACC CardiovasC Imaging. 2018;11:1419-29.

19. Schneeweis C, Qiu J, Schnackenburg B, Berger A, Kelle S, Fleck E, Gebker R. Value of additional strain analysis with feature tracking in dobutamine stress cardiovascular magnetic resonance for detecting coronary artery disease. J Cardiovasc Magnc Reson. 2014;16:72.

20. Williams SG, Cooke GA, Wright DJ, Parsons WJ, Riley RL, Marshall P, Tan LB. Peak exercise cardiac power output; a direct indicator of cardiac function strongly predictive of prognosis in chronic heart failure. Eur Heart J. 2001;22:1496-503.

21. Fincke R, Hochman JS, Lowe AM, Menon V, Slater JN, Webb JG, LeJemtel $\mathrm{TH}$, Cotter G, Investigators S. Cardiac power is the strongest hemodynamic correlate of mortality in cardiogenic shock: a report from the SHOCK trial registry. J Am Coll Cardiol. 2004;44:340-8.

22. Cotter G, Williams SG, Vered Z, Tan LB. Role of cardiac power in heart failure. Curr Opin Cardiol. 2003;18:215-22.

23. Abawi D, Faragli A, Schwarzl M, Manninger M, Zweiker D, Kresoja K-P, Verderber J, Zirngast B, Maechler H, Steendijk P, Pieske B, Post H, Alogna A. Cardiac power output accurately reflects external cardiac work over a wide range of inotropic states in pigs. BMC Cardiovasc Disord. 2019;19:217.

24. Kass DA, Maughan WL. From 'Emax' to pressure-volume relations: a broader view. Circulation. 1988;77:1203-12.

25. Seemann F, Arvidsson P, Nordlund D, Kopic S, Carlsson M, Arheden H, Heiberg E. Noninvasive quantification of pressure-volume loops from brachial pressure and cardiovascular magnetic resonance. Circ Cardiovasc Imaging. 2019;12:e008493.

26. Alogna A, Manninger M, Schwarzl M, Zirngast B, Steendijk P, Verderber J, Zweiker D, Maechler H, Pieske BM, Post H. Inotropic effects of experimental hyperthermia and hypothermia on left ventricular function in pigs-comparison with dobutamine. Crit Care Med. 2016;44:e158-67.

27. Schulz-Menger J, Bluemke DA, Bremerich J, Flamm SD, Fogel MA, Friedrich MG, Kim RJ, von Knobelsdorff-Brenkenhoff F, Kramer CM, Pennell DJ, Plein S, Nagel E. Standardized image interpretation and post processing in cardiovascular magnetic resonance: Society for Cardiovascular Magnetic Resonance (SCMR) board of trustees task force on standardized post processing. J Cardiovasc Magn Reson. 2013;15:35.
28. Carlsson M, Andersson R, Bloch KM, Steding-Ehrenborg K, Mosén H, Stahlberg F, Ekmehag B, Arheden H. Cardiac output and cardiac index measured with cardiovascular magnetic resonance in healthy subjects, elite athletes and patients with congestive heart failure. J Cardiovasc Magn Reson. 2012;14:51.

29. Kelly RP, Ting CT, Yang TM, Liu CP, Maughan WL, Chang MS, Kass DA. Effective arterial elastance as index of arterial vascular load in humans. Circulation. 1992;86:513-21.

30. Rhea IB, Rehman S, Jarori U, Choudhry MW, Feigenbaum H, Sawada SG. Prognostic utility of blood pressure-adjusted global and basal systolic longitudinal strain. Echo Res Pract. 2016;3:17-24.

31. Reichek N, Wilson J, John Sutton M, Plappert TA, Goldberg S, Hirshfeld JW. Noninvasive determination of left ventricular end-systolic stress: validation of the method and initial application. Circulation. 1982;65:99-108.

32. Weaver B, Wuensch KL. SPSS and SAS programs for comparing Pearson correlations and OLS regression coefficients. Behav Res Methods. 2013;45:880-95.

33. Scatteia A, Baritussio A, Bucciarelli-Ducci C. Strain imaging using cardiac magnetic resonance. Heart Fail Rev. 2017;22:465-76.

34. Schuster A, Hor KN, Kowallick JT, Beerbaum P, Kutty S. Cardiovascular magnetic resonance myocardial feature tracking: concepts and clinical applications. Circ Cardiovasc Imaging. 2016;9:e004077.

35. Amzulescu MS, De Craene M, Langet H, Pasquet A, Vancraeynest D, Pouleur AC, Vanoverschelde JL, Gerber BL. Myocardial strain imaging: review of general principles, validation, and sources of discrepancies. Eur Heart J Cardiovasc Imaging. 2019;20:605-19.

36. Weidemann F, Jamal F, Kowalski M, Kukulski T, D'Hooge J, Bijnens B, Hatle $L$, De Scheerder I, Sutherland GR. Can strain rate and strain quantify changes in regional systolic function during dobutamine infusion, B-blockade, and atrial pacing — implications for quantitative stress echocardiography. J Am Soc Echocardiogr. 2002;15:416-24.

37. Yingchoncharoen T, Agarwal S, Popovic ZB, Marwick TH. Normal ranges of left ventricular strain: a meta-analysis. J Am Soc Echocardiogr. 2013;26:185-91.

38. Weiner RB, Weyman AE, Kim JH, Wang TJ, Picard MH, Baggish AL. The impact of isometric handgrip testing on left ventricular twist mechanics. J Physiol. 2012;590:5141-50.

39. Bouchez S, Heyde B, Barbosa D, Vandenheuvel M, Houle H, Wang Y, D'Hooge J, Wouters PF. In-vivo validation of a new clinical tool to quantify three-dimensional myocardial strain using ultrasound. Int J Cardiovasc Imaging. 2016;32:1707-14.

40. Barreiro-Pérez M, Curione D, Symons R, Claus P, Voigt J-U, Bogaert J. Left ventricular global myocardial strain assessment comparing the reproducibility of four commercially available CMR-feature tracking algorithms. Eur Radiol. 2018;28:5137-47.

41. Swoboda PP, Larghat A, Zaman A, Fairbairn TA, Motwani M, Greenwood JP, Plein S. Reproducibility of myocardial strain and left ventricular twist measured using complementary spatial modulation of magnetization. J Magn Reson Imaging. 2014;39:887-94.

42. Schmidt B, Dick A, Treutlein M, Schiller P, Bunck AC, Maintz D, Baeßler B. Intra- and inter-observer reproducibility of global and regional magnetic resonance feature tracking derived strain parameters of the left and right ventricle. Eur J Radiol. 2017:89:97-105.

43. Faragli A, Tanacli R, Kolp C, Lapinskas T, Stehning C, Schnackenburg B, Lo Muzio FP, Perna S, Pieske B, Nagel E, Post H, Kelle S, Alogna A. Cardiovascular magnetic resonance feature tracking in pigs: a reproducibility and sample size calculation study. Int J Cardiovasc Imaging. 2020;36:703-12.

44. Reichek N. Myocardial strain: still a long way to go. Circ Cardiovasc Imaging. 2017. https://doi.org/10.1161/CIRCIMAGING.117.007145.

\section{Publisher's Note}

Springer Nature remains neutral with regard to jurisdictional claims in published maps and institutional affiliations. 